\title{
ANÁLISE DO USO RACIONAL DE HYPERICUM PERFORATUM A PARTIR DO PERFIL DAS PRESCRIÇÕES AVIADAS EM FARMÁCIAS DE ANÁPOLIS - GO
}

\section{Analisys of the rational use of Hypericum perforatum from the profile of the lapsings prepared in Anápolis - GO pharmacies}

\author{
Marcelo G. Rodrigues ${ }^{1,2^{*}}$; Marcelo M. Mendonça ${ }^{1,3}$; Joelma A. M. de Paula ${ }^{1,4}$ \\ ${ }^{1}$ Unidade Universitária de Ciências Exatas e Tecnológicas, Curso de Farmácia, Universidade Estadual de Goiás. \\ BR 153, Km 3. 75110-390. Anápolis - GO, Brasil. \\ ${ }^{2,3}$ Acadêmicos do Curso de Farmácia da Universidade Estadual de Goiás; \\ ${ }^{4}$ Profa das disciplinas Fundamentos de Homeopatia, Redação Técnica e Científica I e Il do Curso de \\ Farmácia/UEG.
}

*Autor para correspondência e-mail: garcez_rodrigues@hotmail.com

Recebido em 06/10/2006 - Aceito em 11/12/2006

RESUMO: O tratamento fitoterápico da depressão pode ser feito, atualmente, por meio do extrato seco padronizado de Hypericum perforatum L., popularmente conhecido como erva-de-são-joão. Há evidências científicas da sua eficácia antidepressiva nos casos leves a moderados e que apontam para um perfil de tolerabilidade superior aos antidepressivos sintéticos. No entanto, há registros na literatura de potenciais interações medicamentosas envolvendo o uso concomitante de $H$. perforatum e outros medicamentos. Isto levanta a necessidade de ações dos profissionais da saúde no sentido de promoverem o uso racional desta erva com vistas à prevenção de complicações aos usuários. Tendo em vista a importância da prescrição médica como uma etapa na cadeia de eventos que contribuem para o uso racional dos fitoterápicos, este trabalho teve como propósito realizar um estudo descritivo das prescrições contendo $H$. perforatum aviadas em farmácias de manipulação da cidade de Anápolis - Goiás, com o intuito de verificar se a comunidade médica desta região tem observado critérios importantes que contribuem para o uso racional deste fitoterápico. Verificou-se que as farmácias analisadas têm aviado prescrições contendo $H$. perforatum em dosagens diárias que guardam conformidade com o que é preconizado na literatura. Além disso, a maior parte das formulações contendo $H$. perforatum foi dispensada na forma isolada (sem associações), sem tarja vermelha contendo os dizeres "VENDA SOB PRESCRIÇÃO MÉDICA" na embalagem de dispensação e na forma de extrato seco padronizado. Concluiuse que a comunidade médica desta cidade vem buscando uma droga alternativa que possa ser utilizada no tratamento ambulatorial da depressão leve a moderada, no entanto a presença de prescrições do $H$. perforatum em associação a outros fitoterápicos representa risco potencial de interação medicamentosa que pode comprometer a efetividade dos tratamentos e colocar em risco a saúde dos usuários.

PALAVRAS-CHAVE: Fitoterapia, depressão, Hypericum perforatum.

ABSTRACT: The herbal medicine treatment of depression can be done currently with Hypericum perforatum L. extract, popularly known as St. John's wort. There are scientific evidences related to its antidepressant efficacy to mild and moderate depression cases that take for a superior tolerance profile than the synthetic antidepressants. However, there are registers in the literature of potential interactions involving the concomitant use of $H$. perforatum and other medicines. This raises the necessity of action of the health professional in the direction to promote the rational use of this herbal medicine with goal to prevent complications to the users. Considering the importance of the medical lapsing as a stage in the chain of events that contribute for the rational use of the $H$. perforatum, it was proposed to accomplish a descriptive study of the $H$. perforatum lapsing prepared in Anápolis City - Goiás pharmacies. It had the intention to verify if the medical community of this region has observed important criterion for the security and effectiveness of this therapy. It was verified that the analyzed pharmacies has prepared lapsing contends $\mathrm{H}$. perforatum in daily dosages that keep conformity with what it is praised in literature. Moreover, most of the formulations that contend $H$. perforatum it was dispensed in the isolated form (without associations), without red border containing the sentence "SELL UNDER MEDICAL LAPSING" in the packing and the standardized dry 
extract form. The study evidenced that the medical community of this city is looking for an alternative drug that can be used in mild to moderate depression ambulatory treatment, however the presence of $H$. perforatum lapsing in association to other herbal medicines represents potential risk of drug interaction that can compromise the effectiveness of the treatments and at risk place the health of the users.

KEYWORDS: Herbal medicines, depression, Hypericum perforatum.

\section{INTRODUÇÃO}

O termo depressão é utilizado rotineiramente para descrever a reação humana normal diante de perda importante. Outras vezes, representa simplesmente o sentimento de tristeza. Em psiquiatria, porém a depressão consiste em transtorno do humor e representa uma síndrome com sintomatologia e sinais bem definidos (MIRANDA-SCIPPA \& OLIVEIRA, 2002). Pode variar de uma condição muito branda, beirando a normalidade, a quadros severos acompanhados por alucinações e delírios (RANG, et al., 2003).

A terapêutica da depressão pode ser feita com acompanhamento psicológico, social, medicamentoso e eletroconvulsoterapia. Essas modalidades podem ser associadas ou isoladas. A evidência sugere que a terapêutica com medicação antidepressiva é mais efetiva, sendo que estudos controlados com placebo demonstram que ela reduz a porcentagem de recidiva (MELEIRO, 2000).

O tratamento farmacológico convencional da depressão leve a moderada classicamente se divide em três grandes grupos: os antidepressivos tricíclicos (ADT) - imipramina, amitriptilina, nortriptilina; os inibidores da monoamina oxidase (IMAO) - iproniazida, fenelzina, amiflamina e os inibidores seletivos da recaptação de serotonina (ISRS) - fluoxetina, venlafaxina e sertralina. De uma maneira geral, estas medicações já foram consagradas como eficazes no tratamento da depressão (MELEIRO, 2000). Mas, apesar do grande avanço nesta área e do desenvolvimento de novas classes de agentes antidepressivos, os profissionais envolvidos com o manejo de pacientes depressivos ainda se defrontam com algumas dificuldades (LIMA, et al., 2004). Dentre as quais se destaca o grande número de efeitos colaterais relacionados ao uso de tais medicações que inviabilizam seu uso, gerando uma taxa de abandono de tratamento que varia de $7,7 \%$ a $16,2 \%$ (MELEIRO, 2000). A comunidade médica tem sentido, portanto, a necessidade de uma droga alternativa que seja eficaz, com menos efeitos colaterais e que possa ser utilizada com segurança no tratamento ambulatorial da depressão leve a moderada (MELEIRO, 2000).

A planta Hypericum perforatum L., ou erva-de-são-joão como é popularmente conhecida, tem sido utilizada há séculos na medicina popular como antidepressivo e regulador dos distúrbios do sono, sobretudo na Europa (ALONSO, 1998). Na Alemanha, onde apresenta sua maior aceitação, é o antidepressivo mais utilizado e representa mais de $25 \%$ do total dos antidepressivos prescritos e quatro vezes mais do que a quantidade utilizada de fluoxetina (BAHLS, 2001). A despeito de seu uso comum e de sua provável eficácia, seu mecanismo de ação ainda permanece incerto (SINGH, 2005). Além disso, as potenciais interações medicamentosas em podem ocorrer quando este fitoterápico é administrado concomitantemente com medicamentos prescritos para o tratamento de desordens comuns (CORDEIRO, et al., 2005; KARHOVA, et al., 2000; MURPHY, 2002; SINGH, 2005; WOLD, et al., 2005) levantam a necessidade do empreendimento de ações executadas por profissionais da área da saúde, em especial médicos e farmacêuticos, no sentido de promover o seu uso racional (RATES, 2001).

Após a aprovação da Política Nacional de Práticas Integrativas e Complementares (PNPIC) no Sistema Único de Saúde, que democratiza o acesso da população à Fitoterapia por considerar que "tal abordagem incentiva o desenvolvimento comunitário, a solidariedade e a participação social" (BRASIL, 2006a), as autoridades de saúde e o governo federal expressaram a preocupação em garantir à população brasileira o acesso seguro e o uso racional desses produtos, aprovando em junho de 2006 a Política Nacional de Plantas Medicinais e Fitoterápicos e instituindo um Grupo de Trabalho com a finalidade de elaborar o Programa Nacional de Plantas Medicinais e Fitoterápicos (BRASIL, 2006b).

Diversos alertas têm surgido na literatura, científica e leiga, no sentido de incentivar a comunidade médica à utilização de fitoterápicos como uma possibilidade real de tratamento. Não se deve olvidar, entretanto, que esta utilização deve ponderar sobre a dosagem, a forma de dispensação, os efeitos benéficos, os potenciais efeitos tóxicos e as interações medicamentosas, a fim de assegurar o uso racional dos mesmos (RATES, 2001).

Tendo em vista o fato de que a prescrição médica configura-se como uma etapa importante na cadeia de eventos que contribuem para o uso racional dos fitoterápicos (RATES, 2001), este trabalho teve como propósito realizar um estudo descritivo das prescrições contendo $H$. perforatum aviadas em farmácias de manipulação da cidade de Anápolis - Goiás, nos anos de 2004 e 2005, em comparação às prescrições de antidepressivos sintéticos, com o intuito de verificar se a comunidade médica desta região tem observado critérios importantes que contribuem para o uso racional deste fitoterápico, tais como, forma de dispensação, dosagem adequada e interações medicamentosas que podem ocorrer com a sua utilização. 


\section{MATERIAL E MÉTODOS}

De acordo com o Conselho Regional de Farmácia do Estado de Goiás (2006), existem, atualmente, 10 farmácias de manipulação registradas e em funcionamento no município de Anápolis. Todas elas foram procuradas para colaborarem com esta pesquisa, porém, apenas 3 concordaram em disponibilizar as informações necessárias, o que correspondeu a uma amostra de $30 \%$ da população configurada. Os dados destas 3 farmácias foram coletados no período de janeiro de 2004 a dezembro de 2005.

Para a coleta de dados elaborou-se um relatório de dispensação que foi entregue ao farmacêutico técnico responsável de cada farmácia. Estes farmacêuticos, por possuírem acesso aos livros de registros e/ou sistemas informatizados de tais farmácias puderam preencher estes relatórios prestando informações quanto à quantidade de receitas com formulações contendo o Hypericum perforatum e contendo medicamentos não-fitoterápicos (antidepressivos sintéticos) aviadas, bem como quanto à ocorrência de prescrições do $H$. perforatum em associação com outras substâncias, a forma (extrato seco padronizado ou droga pulverizada) e dosagem pela qual o Hipérico foi prescrito e dispensado. Além disso, convém ressaltar que, pelo fato de muitas farmácias de manipulação interpretarem a Resolução Específica n. 357 da ANVISA (BRASIL, 2002b) como extensiva ao medicamento magistral verificou-se também, a presença ou ausência de tarja vermelha contendo os dizeres "VENDA SOB PRESCRIÇÃO MÉDICA" na embalagem de dispensação.

$\mathrm{Na}$ elaboração do relatório de dispensação selecionou-se princípios ativos sintéticos com ação predominantemente antidepressiva pertencentes a 3 grandes grupos: antidepressivos tricíclicos, antidepressivos inibidores da monoamina oxidase e antidepressivos de segunda geração. No momento da entrega do relatório ao farmacêutico técnico, o mesmo foi questionado sobre a utilização de algum outro medicamento sintético com ação antidepressiva disponível e dispensado na farmácia e que não constasse no relatório de dispensação cuja resposta foi negativa.

\section{RESULTADOS E DISCUSSÃO}

A fim de preservar a identidade das farmácias de manipulação envolvidas neste estudo elas foram referidas apenas como Farmácia 1, Farmácia 2 e Farmácia 3.

A Tabela 1 enumera as prescrições de antidepressivos sintéticos e Hypericum perforatum dispensadas, durante os anos de 2004 e 2005, nas 3 farmácias de manipulação de Anápolis analisadas neste estudo.

Tabela 1 - Número de prescrições de Antidepressivos sintéticos e Hypericum perforatum nos anos de 2004 e 2005 em farmácias de manipulação de Anápolis.

\begin{tabular}{ccccc}
\hline \multicolumn{2}{c}{ Antidepressivos Sintéticos } & \multicolumn{3}{c}{ Número de Prescrições } \\
\hline Nome Genérico & Grupo Farmacológico & Farmácia 1 & Farmácia 2 & Farmácia 3 \\
Amitriptilina & & & & \\
Bupropiona & ADT & 190 & 310 & 1852 \\
Citalopram & IRND & - & 12 & 42 \\
Clomipramina & ISRS & 10 & 12 & 290 \\
Fluoxetina & ADT & - & 06 & - \\
Fluvoxamina & ISRS & 1300 & 2529 & 3758 \\
Imipramina & ISRS & - & - & - \\
Maprotilina & ADT & - & 20 & 50 \\
Moclobemida & ISRS & - & - & - \\
Nortriptilina & IMAO-A & - & - & - \\
Paroxetina & ADT & 50 & 73 & 668 \\
Reboxetina & ISRS & 36 & 42 & 581 \\
Sertralina & ISRN & - & - & - \\
Tranilcipromina & ISRS & 399 & 412 & 1924 \\
Venlafaxina & IMAO não-seletivo & - & - & - \\
H. perforatum & IRSN & 54 & - & 250 \\
\hline
\end{tabular}

Legenda: $\mathrm{ADT}=$ Antidepressivo tricíclico; IMAO-A = Inibidor da monoamina oxidase tipo A; IMAO não-seletivo = Inibidor da monoamina oxidase não-seletivo para os subtipos de monoamina A e B; IRND = Inibidor da recaptação de noradrenalina e dopamina; IRSN = Inibidor da recaptação de serotonina e noradenalina; ISRN = Inibidor seletivo da recaptação de noradrenalina; ISRS = Inibidor seletivo da recaptação de serotonina.

De acordo com a Tabela 1, verificou-se que o maior número de prescrições nas farmácias de manipulação de Anápolis, no período de janeiro de 2004 a dezembro de 2005, ocorreu quanto à classe dos antidepressivos de $2^{\mathrm{a}}$ geração (IRND; IRSN; ISRN e ISRS), perfazendo um total de $88,22 \%$; $88,02 \%$ e $72,7 \%$ das formulações 
dispensadas de antidepressivos sintéticos nas Farmácias 1,2 e 3, respectivamente. O segundo grupo de antidepressivos mais prescrito foi representado pelos antidepressivos tricíclicos que, nas farmácias de manipulação 1, 2 e 3, respectivamente, perfizeram um total de 11,77\%; 11,97\% e 27,29\% das formulações dispensadas em relação à prescrição de antidepressivos sintéticos.

A Figura 1 ilustra um panorama das prescrições nas farmácias de manipulação de Anápolis das diferentes classes de antidepressivos sintéticos.

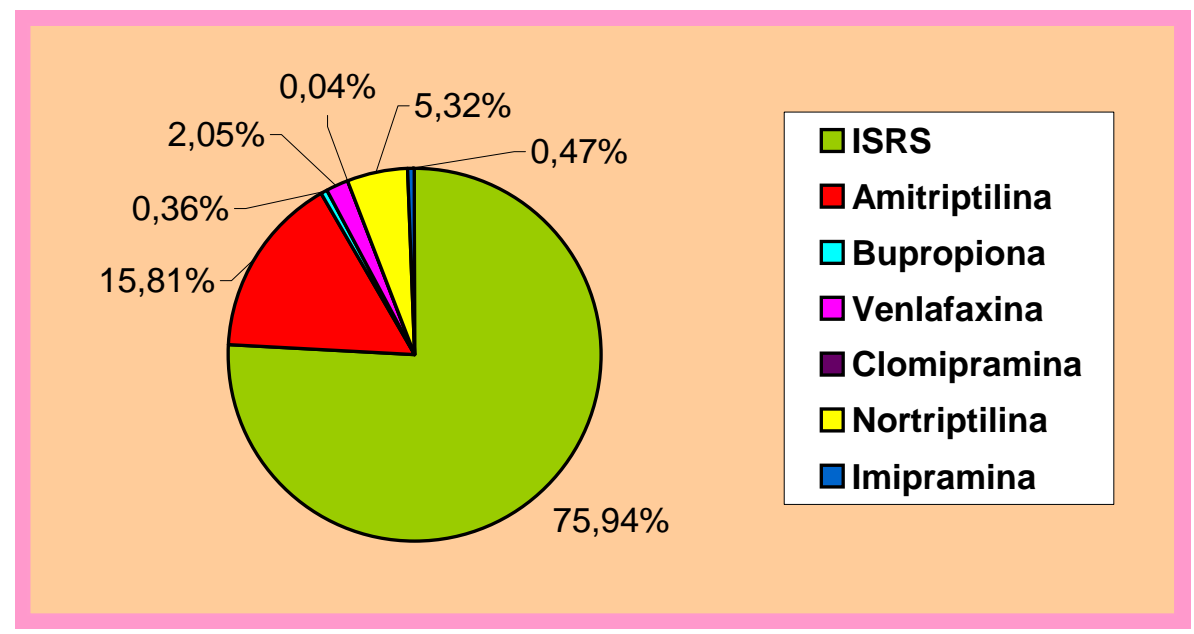

Figura 1 - Panorama geral de prescrições das diferentes classes de antidepressivos sintéticos nas farmácias de manipulação de Anápolis durante os anos de 2004 e 2005.

Legenda: ISRS = Inibidor seletivo da recaptação de serotonina.

A alta aceitabilidade médica e a freqüente prescrição dos antidepressivos inibidores seletivos da recaptação de serotonina podem ser justificadas pelas vantagens que esta classe apresenta em relação aos antidepressivos convencionais. Apesar de apresentarem efeitos colaterais freqüentes como distúrbios gastrointestinais, cefaléia, agitação, insônia e redução na conduta sexual, estes medicamentos possuem meiasvidas prolongadas e, portanto, permitem administração em dose única diária, contribuindo para maior adesão ao tratamento. Possuem baixo risco de toxicidade, se ingeridos em altas doses, e têm menos efeitos anticolinérgicos que os antidepressivos tricíclicos, além de não requererem dieta restrita em tiramina como os IMAO (CASS, 1999; KERWIN, et al., 2004; MIRANDA-SCIPPA \& OLIVEIRA, 2002; RANG, et al., 2003; WANNMACHER, 2004). Somase a isto ainda o fato de que tais medicamentos podem ser utilizados, além do tratamento da depressão, no tratamento do transtorno obsessivo-compulsivo (TOC), no tratamento crônico do transtorno do pânico, na fobia social e na bulimia nervosa (MIRANDA-SCIPPA \& OLIVEIRA, 2002; PITLIUK, 2005).

Os antidepressivos tricíclicos constituem um grupo importante de antidepressivos de uso clínico. Contudo, eles estão longe do ideal na prática terapêutica, pois são necessárias substâncias que ajam mais rápida e confiavelmente e produzam menor quantidade de efeitos colaterais (RANG, et al., 2003), fator este provavelmente relacionado à menor porcentagem de prescrições dos ADT $(21,64 \%)$ em relação aos antidepressivos de $2^{\mathrm{a}}$ geração $(78,35 \%)$ nas farmácias analisadas, conforme indica a Figura 1 . Um fator que explica sua prescrição é o preço visto que são consideravelmente mais baratos do que os inibidores seletivos da recaptação de serotonina uma vez que as suas patentes já expiraram e, portanto, podem ser manipulados (CASS, 1999; WANNMACHER, 2004).

A Figura 2 ilustra de forma comparativa a porcentagem total de prescrições de antidepressivos sintéticos e H. perforatum para cada uma das 3 farmácias analisadas neste estudo. 


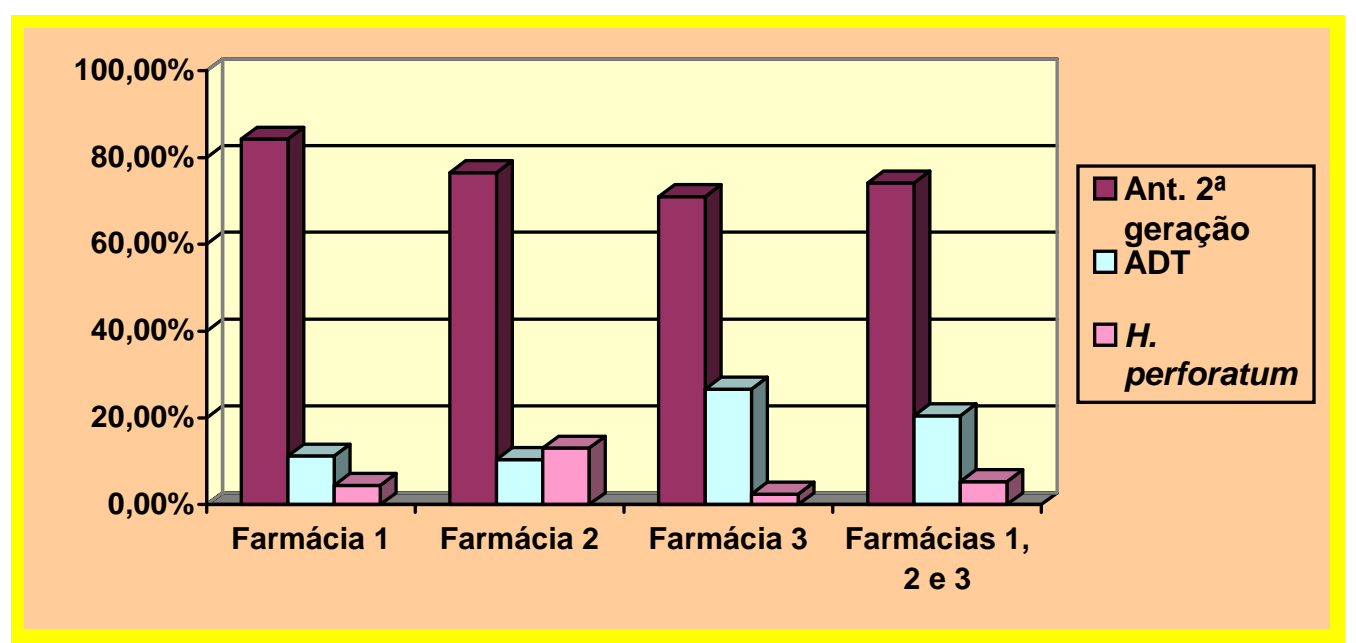

Figura 2 - Comparativo entre a porcentagem total de prescrições de antidepressivos sintéticos e H. perforatum nas farmácias de manipulação de Anápolis durante os anos de 2004 e 2005.

Legenda: Ant. $2^{\mathrm{a}}$ geração = Antidepressivos de segunda geração; ADT = Antidepressivos tricíclicos.

Conforme demonstra a Figura 2, nos anos de 2004 e 2005, o percentual de formulações com $H$. perforatum dispensadas em comparação aos antidepressivos sintéticos foi de $4,49 \%, 13,06 \%$ e $2,42 \%$ das prescrições de antidepressivos nas farmácias de manipulação 1, 2 e 3, respectivamente.

As prescrições de $H$. perforatum nas farmácias analisadas perfizeram um total de 5,36\% em comparação a 94,63\% dos antidepressivos sintéticos. Tal porcentagem é justificável uma vez que os antidepressivos sintéticos, de um modo geral, além de serem utilizados para o tratamento da depressão, podem também ser administrados para enxaquecas, tensão pré-menstrual, dores crônicas, reumáticas e oncológicas, enurese noturna em crianças, além de casos mais específicos de transtorno obsessivo-compulsivo (TOC), anorexia, bulimia nervosa e síndrome do pânico (MIRANDA-SCIPPA, 2002; PITLIUK, 2005; WANNMACHER, 2004). Já os estudos clínicos envolvendo o $H$. perforatum comprovaram sua eficácia apenas em casos de depressão leve a moderada (BAHLS, 2001; BALLONE, 2005; CAMPOS, 2003; CASS, 1999; CORDEIRO, et al., 2005; D'IPPOLITO, et al., 2005; LINDE, et al., 1996; MANNEL, 2004; MELEIRO, 2000; SCHULZ, et al., 2002; TAVARES JÚNIOR, 1999; WANNMACHER, 2004; WHO, 2002). Este estudo não fez distinção entre os antidepressivos prescritos e a sua indicação clínica.

Todavia, apesar da baixa porcentagem de prescrições do $H$. perforatum em relação à porcentagem total de prescrições contendo antidepressivos sintéticos, verifica-se que o fitoterápico foi manipulado em todas as farmácias analisadas. A porcentagem total de prescrições para formulações contendo $H$. perforatum (13,06\%), na farmácia 2, foi superior à dos antidepressivos tricíclicos (10,40\%) e inferior apenas ao número de prescrições $(64,36 \%)$ da fluoxetina.

As Tabelas 2, 3 e 4 especificam informações quanto à posologia, forma e embalagem para dispensação e eventuais associações das formulações prescritas de $H$. perforatum para cada farmácia analisada neste estudo.

Tabela 2 - Especificações das formulações farmacêuticas dispensadas contendo H. perforatum nos anos de 2004 e 2005 na Farmácia 1.

\begin{tabular}{|c|c|c|c|c|c|}
\hline \multirow{2}{*}{$\begin{array}{l}\text { Número de } \\
\text { Prescrições }\end{array}$} & \multicolumn{2}{|c|}{ "Forma de uso" } & \multirow[t]{2}{*}{ Associações } & \multirow[t]{2}{*}{$\begin{array}{c}\text { Forma de } \\
\text { dispensação (extrato } \\
\text { seco padronizado / } \\
\text { droga pulverizada) }\end{array}$} & \multirow{2}{*}{$\begin{array}{c}\text { Embalagem para } \\
\text { dispensação } \\
\text { (presença ou } \\
\text { ausência da tarja } \\
\text { vermelha) } \\
\end{array}$} \\
\hline & Dosagem & Posologia & & & \\
\hline 06 & $100 \mathrm{mg}$ & $3 \times$ ao dia & - & Ext. seco padroniz. & Presença \\
\hline 07 & 150 mg & $3 \times$ ao dia & - & Ext. seco padroniz. & Presença \\
\hline 09 & $200 \mathrm{mg}$ & $3 \times$ ao dia & - & Ext. seco padroniz. & Presença \\
\hline 14 & $250 \mathrm{mg}$ & $2 \times$ ao dia & - & Ext. seco padroniz. & Presença \\
\hline 16 & $300 \mathrm{mg}$ & $3 \times$ ao dia & - & Ext. seco padroniz. & Presença \\
\hline 10 & $350 \mathrm{mg}$ & $2 \times$ ao dia & - & Ext. seco padroniz. & Presença \\
\hline 09 & $300 \mathrm{mg}$ & $1 \times$ ao dia & Valeriana (150 mg) & Ext. seco padroniz. & Presença \\
\hline 03 & $400 \mathrm{mg}$ & $1 \times$ ao dia & Valeriana (300 mg) & Ext. seco padroniz. & Presença \\
\hline 03 & $250 \mathrm{mg}$ & $2 \times$ ao dia & Melissa (500 mg) & Ext. seco padroniz. & Presença \\
\hline 05 & $350 \mathrm{mg}$ & $2 \times$ ao dia & Melissa (500 mg) & Ext. seco padroniz. & Presença \\
\hline 08 & $300 \mathrm{mg}$ & $2 \times$ ao dia & Kava-Kava (100 mg) & Ext. seco padroniz. & Presença \\
\hline 06 & $350 \mathrm{mg}$ & $2 \times$ ao dia & Kava-Kava (150 mg) & Ext. seco padroniz. & Presença \\
\hline
\end{tabular}


Legenda: Ext. seco padroniz. $=$ Extrato seco padronizado de Hypericum perforatum .

Tabela 3 - Especificações das formulações farmacêuticas dispensadas contendo H. perforatum nos anos de 2004 e 2005 na Farmácia 2.

\begin{tabular}{|c|c|c|c|c|c|}
\hline \multirow{2}{*}{$\begin{array}{l}\text { Número de } \\
\text { Prescrições }\end{array}$} & \multicolumn{2}{|c|}{ “Forma de uso" } & \multirow[t]{2}{*}{ Associações } & \multirow{2}{*}{$\begin{array}{c}\text { Forma de } \\
\text { dispensação (extrato } \\
\text { seco padronizado / } \\
\text { droga pulverizada) }\end{array}$} & \multirow{2}{*}{$\begin{array}{l}\text { Embalagem para } \\
\text { dispensação } \\
\text { (presença ou } \\
\text { ausência da tarja } \\
\text { vermelha) }\end{array}$} \\
\hline & Dosagem & Posologia & & & \\
\hline 30 & $350 \mathrm{mg}$ & $3 \times$ ao dia & Passiflora (100 mg) & Droga pulverizada & Ausência \\
\hline 23 & 400 mg & $3 \times$ ao dia & - & Droga pulverizada & Ausência \\
\hline 71 & $450 \mathrm{mg}$ & $3 \times$ ao dia & - & Droga pulverizada & Ausência \\
\hline 130 & $500 \mathrm{mg}$ & $3 \times$ ao dia & - & Droga pulverizada & Ausência \\
\hline 64 & $250 \mathrm{mg}$ & $3 \times$ ao dia & - & Ext. seco padroniz. & Ausência \\
\hline 43 & 300 mg & $3 \times$ ao dia & Kava-Kava (100 mg) & Ext. seco padroniz. & Ausência \\
\hline 27 & 300 mg & $2 \times$ ao dia & Kava-Kava (150 mg) & Ext. seco padroniz. & Ausência \\
\hline 125 & 350 mg & $2 \times$ ao dia & - & Ext. seco padroniz. & Ausência \\
\hline
\end{tabular}

Legenda: Ext. seco padroniz. $=$ Extrato seco padronizado de Hypericum perforatum .

Tabela 4 - Especificações das formulações farmacêuticas dispensadas contendo H. perforatum nos anos de 2004 e 2005 na Farmácia 3.

\begin{tabular}{|c|c|c|c|c|c|}
\hline \multirow{2}{*}{$\begin{array}{l}\text { Número de } \\
\text { Prescrições }\end{array}$} & \multicolumn{2}{|c|}{ "Forma de uso" } & \multirow[t]{2}{*}{ Associações } & \multirow{2}{*}{$\begin{array}{c}\text { Forma de } \\
\text { dispensação (extrato } \\
\text { seco padronizado / } \\
\text { droga pulverizada) }\end{array}$} & \multirow{2}{*}{$\begin{array}{l}\text { Embalagem para } \\
\text { dispensação } \\
\text { (presença ou } \\
\text { ausência da tarja } \\
\text { vermelha) }\end{array}$} \\
\hline & Dosagem & Posologia & & & \\
\hline 91 & $150 \mathrm{mg}$ & $2 \times$ ao dia & - & Ext. seco padroniz. & Presença \\
\hline 23 & 180 mg & $3 \times$ ao dia & - & Ext. seco padroniz. & Presença \\
\hline 51 & $200 \mathrm{mg}$ & $2 \times$ ao dia & - & Ext. seco padroniz. & Presença \\
\hline 37 & $300 \mathrm{mg}$ & $2 \times$ ao dia & - & Ext. seco padroniz. & Presença \\
\hline 06 & $150 \mathrm{mg}$ & $2 \times$ ao dia & $\begin{array}{c}\text { Tiamina }(30 \mathrm{mg}) \\
\text { Piridoxina }(30 \mathrm{mg}) \\
\text { Cianocobalamina }(50 \\
\text { mcg) } \\
\text { Ácido Fólico }(2,5 \mathrm{mg})\end{array}$ & Ext. seco padroniz. & Presença \\
\hline 04 & $150 \mathrm{mg}$ & $2 \times$ ao dia & Kava-Kava (150 mg) & Ext. seco padroniz. & Presença \\
\hline 05 & $350 \mathrm{mg}$ & $1 \times$ ao dia & Kava-kava (250 mg) & Ext. seco padroniz. & Presença \\
\hline 06 & $100 \mathrm{mg}$ & $3 \times$ ao dia & Passiflora (100 mg) & Ext. seco padroniz. & Presença \\
\hline 08 & $300 \mathrm{mg}$ & $1 \times$ ao dia & Passiflora (200 mg) & Ext. seco padroniz. & Presença \\
\hline 03 & $250 \mathrm{mg}$ & $3 \times$ ao dia & Ginkgo biloba (80mg) & Ext. seco padroniz. & Presença \\
\hline
\end{tabular}

Legenda: Ext. seco padroniz. = Extrato seco padronizado de Hypericum perforatum.

De acordo com a Tabela 2 verificou-se que na farmácia 1 a dosagem diária do extrato seco padronizado de H. perforatum mais prescrita foi a de $700 \mathrm{mg}$. As dosagens diárias de $500 \mathrm{mg}, 600 \mathrm{mg}, 900 \mathrm{mg}$ e $300 \mathrm{mg}$ tiveram um número intermediário de prescrições e o menor número destas ocorreu com as dosagens diárias de 450 e 400 mg. Na farmácia 2, conforme indica a Tabela 3, a dosagem diária de 700 mg foi responsável pelo maior número de dispensações seguida, de forma decrescente do número de formulações dispensadas, pelas dosagens diárias de $750 \mathrm{mg}, 600 \mathrm{mg}$ e $900 \mathrm{mg}$. A dosagem diária de $300 \mathrm{mg}$ liderou o número de prescrições do extrato seco padronizado de $H$. perforatum na Farmácia 3 de acordo com a Tabela 4 . O número de prescrições intermediário ficou com as dosagens diárias de 400 mg, 540 mg e 600 mg. As dosagens diárias de 350 mg e 750 mg representaram o menor número de prescrições. Nas farmácias de manipulação analisadas, todas as prescrições envolveram dosagens diárias que variaram de $300 \mathrm{mg}$ a $900 \mathrm{mg}$. Esta faixa de dosagem mantém conformidade com o que é preconizado pela World Health Organization (2002) que recomenda a dose mínima de $300 \mathrm{mg}$ de extrato diariamente, com uma dose normal na faixa de 500-900 mg/dia.

Apenas uma única farmácia (2) dispensou formulações com $H$. perforatum na forma de droga pulverizada. De acordo com a Tabela 3 nota-se que das 254 formulações dispensadas na forma de pó as dosagens diárias de $1050 \mathrm{mg}, 1200 \mathrm{mg}, 1350 \mathrm{mg}$ e $1500 \mathrm{mg}$ foram responsáveis, respectivamente, por 11,81\%, 9,05\%, 27,95\% e $51,18 \%$ do número total de formulações. Dosagens estas que estão conforme o que determina a comissão $\mathrm{E}$ da 
Agência Federal de Saúde Alemã. Para a droga vegetal em pó, a dose diária recomendada varia entre 1-3 g/dia (CASS, 1999; SCHULZ, et al., 2002).

Foi constatado também que o maior número de dispensações de formulações contendo $H$. perforatum deu-se na farmácia 2 (513 formulações dispensadas em comparação com 96 na farmácia 1 e 234 na farmácia 3 ) e a mesma foi a única a dispensar o Hypericum tanto na forma em pó como na forma de extrato seco padronizado. O maior número de dispensações na farmácia 2 pode estar relacionada ao fato de que a droga em pó do $H$. perforatum é mais acessível à população devido ao seu custo relativamente menor quando comparado com o extrato seco padronizado da erva. Na referida farmácia, por exemplo, o custo de 60 cápsulas de 350 mg do extrato seco padronizado da droga chega a ser mais que o dobro do custo de uma formulação equivalente da droga em pó. Entretanto, a eficácia da droga em pó do $H$. perforatum não pode ser comparada à do extrato seco padronizado, pois não há padronização da concentração de substâncias consideradas essencialmente importantes para a ação antidepressiva, como a hipericina e a hiperforina (BAHLS, 2001; SCHULZ, et al., 2002). Um extrato de Hypericum em metanol ou etanol deve conter aproximadamente 0,1-0,3\% de hipericinas e 1-6\% de hiperforina para que seja eficaz (SCHULZ, et al., 2002). Tal observação é importante uma vez que nem todas as prescrições médicas para a manipulação de formulações contendo $H$. perforatum apresentam especificação quanto à apresentação na forma de droga pulverizada ou extrato seco padronizado.

A associação da erva-de-são-joão com várias outras ervas pode apresentar efeitos terapêuticos valiosos, uma vez que a ação terapêutica das ervas medicinais pode, mediante geralmente à grande quantidade de substâncias em sua composição química, atuar em sinergismo para reforçar os efeitos positivos de cada uma (CASS, 1999). Por outro lado, estas associações devem ser vistas com muita cautela, pois apesar deste estudo não ter investigado a utilização de outros medicamentos pelo paciente no ambiente domiciliar, o uso concomitante de outras ervas e/ou medicamentos e $H$. perforatum propicia um potencial de interações físico-químicas e/ou farmacocinéticas e/ou farmacodinâmicas que pode contribuir para a falha terapêutica, para o aparecimento de efeitos tóxicos e para a progressão da doença (CORDEIRO, et al., 2005; GRÖNING, et al., 2003; KARHOVA, et al., 2000; MURPHY, 2002; SINGH, 2005; WOLD, et al., 2005).

Nas farmácias de manipulação analisadas o maior número de associações ocorreu entre o $H$. perforatum e Piper methysticum G. Forst. (Kava-Kava) que somou 15,79\% das formulações dispensadas. A raiz da planta $P$. methysticum é usualmente combinada com o H. perforatum para pacientes com um misto de depressão e estados de ansiedade nervosa, tensão e agitação. Nas farmácias de manipulação de Anápolis foram prescritas posologias (Tabelas 2, 3 e 4) que envolviam a dosagem de $100 \mathrm{mg} 2$ vezes ao dia ou 3 vezes ao dia; e $150 \mathrm{mg} 2$ vezes ao dia do extrato seco padronizado (contendo $30 \%$ de Kavalactonas) de $P$. methysticum em associação com $H$. perforatum. Essas são dosagens usuais para os estados de ansiedade. Foi também verificada a dosagem de 250 mg 1 vez ao dia (Tabela 4), posologia esta mais utilizada em casos de insônia, cuja indicação é de 200 a 300 mg do extrato seco padronizado (30\% cavalactonas) de $P$. methysticum antes de dormir (CASS, 1999; D'IPPOLITO, et al., 2005; SCHULZ, et al., 2002).

A associação destes fitoterápicos é questionável. As kavalactonas, princípio ativo ao qual se atribui as atividades farmacológicas da Kava-Kava, é um potente inibidor de várias enzimas do sistema citocromo $P$ 450, 0 que sugere um elevado potencial para causar interações farmacocinéticas com outras drogas e fitoterápicos que são metabolizados pelo mesmo sistema enzimático. Algumas kavalactonas têm demonstrado possuir ações farmacológicas tais como bloqueadora de receptores GABAérgicos e de canais de cálcio e sódio, o que pode levar ao aparecimento de interações farmacodinâmicas com outras substâncias que possuem propriedades farmacológicas similares. Além disso, diversos casos de toxicidade hepática grave foram relacionados à utilização de produtos à base de extratos de Kava-Kava. No que se refere ao $\mathrm{H}$. perforatum, sabe-se que seus princípios ativos (hipericina, pseudohipericina e hiperforina) são indutores do sistema de transporte da Glicoproteína $P$ intestinal e de várias enzimas do sistema citocromo P 450, o que poderia provocar a alteração na distribuição e disponibilidade de drogas que utilizam estas vias (CORDEIRO, et al., 2005; SINGH, 2005).

Estados de agitação nervosa e distúrbios do sono são considerados como as indicações tradicionais para o uso de preparações feitas com Valeriana officinalis L., Melissa officinalis L. e Passiflora incarnata L., pois estas contêm princípios ativos com finalidade ansiolítica, o que justificaria a utilização destas plantas em associação ao H. perforatum (SILVEIRA, 2002). Embora haja alguns resultados preliminares positivos, não existem evidências claras da eficácia destas abordagens fitoterápicas comumente empregadas no tratamento de transtornos ansiosos. Eventuais benefícios de um efeito placebo podem não ser duradouros, havendo recorrência dos sintomas, o que contribui para a perda de confiança no médico. Além disso, a maior parte dessas associações não foi submetida à avaliação adequada de eventuais efeitos adversos (WANNMACHER, 2004).

A posologia de Ginkgo biloba L. prescrita nas farmácias de manipulação de Anápolis (Tabela 4) é usualmente utilizada para arteriopatias crônicas como corretivo da diminuição intelectual, especialmente em pessoas idosas, por ativar o metabolismo energético das células nervosas aumentando a captação de glicose e normalizando o consumo de oxigênio o que favorece a circulação cerebral (CASS, 1999; D'IPPOLITO, et al., 2005). Há interações em potencial que tornam irracional a utilização concomitante de G. biloba e $H$. perforatum. Deve-se considerar que o $H$. perforatum pode diminuir a biodisponibilidade de muitas drogas, como discutido anteriormente, o que pode acarretar em diminuição ou ausência de efeitos de medicamentos ou fitoterápicos utilizados concomitantemente a ele. Além disso, o G. biloba pode levar à diminuição de hormônios sexuais 
endógenos tais como, estrógenos e progesterona, e sabe-se que o aparecimento de sangramento e gravidez não planejada em mulheres que fazem uso de contraceptivos esteroides estão associados ao uso concomitante de $H$. perforatum (MURPHY, 2002; WOLD, et al., 2005).

A associação da erva-de-são-joão com vitaminas, tais como: tiamina, piridoxina, cianocobalamina e ácido fólico, dá-se pelo fato de que estas constituem importantes co-fatores para o tratamento da depressão. Tal importância está relacionada à ação das vitaminas como coenzimas em sistemas enzimáticos envolvendo aminoácidos para a síntese de neurotransmissores e/ou degradação de metabólitos; participam de sistemas enzimáticos relacionados com o metabolismo de carboidratos e são importantes para a formação de eritrócitos cuja deficiência pode ocasionar variações de humor (CASS, 1999; SACRAMENTO \& SILVA, 2002).

Recentes relatos de agências regulatórias de produtos pelo mundo têm alertado para as importantes interações entre os produtos à base de $H$. perforatum e medicamentos prescritos, tais como: ciclosporina, digoxina, contraceptivos orais, teofilina, varfarina, indinavir e potencialmente com diversos outros medicamentos vendidos sob prescrição médica (BRASIL, 2002b; OMS, 2000). Uma vez que a cultura popular é baseada na crença de que "produto natural não faz mal", é importante que médicos e farmacêuticos questionem e alertem seus pacientes sobre o uso concomitante de $H$. perforatum e os medicamentos supra mencionados (ANDREATINI, 2005; CORDEIRO, et al., 2005). Faz-se necessário enfatizar a importância da conscientização da equipe de profissionais envolvidos nos processos de prescrição, dispensação e administração dos medicamentos para a utilização correta e racional de fitoterápicos.

Em decorrência disto, a Agência Nacional de Vigilância Sanitária - Anvisa resolveu por meio da publicação das Resoluções Específicas - RE n. 356 e 357, de 28 de fevereiro de 2002 promover uma medida de interesse sanitário de apreensão dos produtos farmacêuticos à base de Erva-de-são-joão e kava-kava que não possuam tarja vermelha contendo os dizeres "VENDA SOB PRESCRIÇÃO MÉDICA", bem como os que não possuam registro na Anvisa. Os produtos farmacêuticos sem o devido padrão de rotulagem passaram a ser apreendidos imediatamente e a empresa autuada (BRASIL, 2002a; BRASIL, 2002b).

É necessário ressaltar que tais resoluções aplicam-se apenas aos produtos industrializados uma vez que estes devem obrigatoriamente apresentar registro na referida autoridade sanitária. Já para as formulações magistrais não há exigência da presença da tarja vermelha contendo os dizeres "VENDA SOB PRESCRIÇÃO MÉDICA" na embalagem de dispensação uma vez que as mesmas não necessitam de registro junto à ANVISA, levando-se em consideração que toda formulação magistral só é manipulada e dispensada nas farmácias mediante prescrição médica.

Neste contexto, foi observado que as farmácias de manipulação apresentaram interpretações diferentes quanto às resoluções específicas n. 356 e 357 da Anvisa (BRASIL 2002a; BRASIL, 2002b) uma vez que foi verificado que todas as formulações contendo $H$. perforatum e/ou $P$. methysticum foram dispensadas, nas farmácias 1 e 3 (Tabelas 2 e 4), com presença da referida tarja vermelha na embalagem de dispensação. Tal procedimento pode ter sido adotado pelas farmácias objetivando não contrariar a legislação vigente ao interpretarem produto farmacêutico como sinônimo de fórmula magistral. Já, provavelmente baseando a interpretação da legislação nesta distinção, durante o período analisado, nenhuma formulação à base de Hipérico e/ou Kava-Kava foi dispensada na farmácia 2 (Tabela 3) contendo tarja vermelha.

A não obrigatoriedade da tarja vermelha e a ausência da necessidade de retenção de receita facilitam o acesso da população aos produtos à base do $H$. perforatum e $P$. methysticum por meio das farmácias de manipulação. Faz-se necessário, portanto, enfatizar o papel do profissional farmacêutico como responsável pela dispensação do medicamento. Este profissional, nas farmácias de manipulação, não deve permitir a obtenção de formulações à base de $H$. perforatum e $P$. methysticum pela população sem a devida prescrição médica. Tal postura deve ser assumida a fim de se evitar o consumo irracional e indiscriminado de tais formulações que podem ocasionar casos de hepatotoxicidade por Kava-Kava, bem como aumentar o risco de interações medicamentosas clinicamente importantes entre o Hipérico e diversas outras drogas.

Portanto, a atitude mais adequada em relação ao uso de fitoterápicos é considerá-los com o mesmo rigor com que se lida com os medicamentos sintéticos, visando diminuir a freqüência e os riscos de automedicação que pode acarretar intoxicações, interações potencialmente perigosas, emprego de tratamento de eficácia não comprovada no lugar de uma terapêutica eficaz e a não procura de um profissional especializado na área da saúde mental.

\section{CONCLUSÂO}

A coleta de dados para análise e constituição dos resultados baseou-se na veracidade das informações disponibilizadas pelos farmacêuticos das farmácias de manipulação analisadas neste estudo e, portanto, representa uma limitação metodológica bem conhecida. Entretanto, estes dados tornam possível a elaboração das seguintes considerações finais:

A amostra de farmácias de manipulação de Anápolis analisada neste estudo tem aviado prescrições contendo $H$. perforatum em dosagens diárias que guardam conformidade com o que é preconizado na literatura (WHO, 2002). 
Foi também observado que a maior parte das formulações à base de $H$. perforatum foram dispensadas sem tarja vermelha contendo os dizeres "VENDA SOB PRESCRIÇÃO MÉDICA" na embalagem de dispensação em comparação com as formulações dispensadas apresentando a referida tarja. Tal ocorrência pode estar relacionada a diferentes interpretações das Resoluções Específicas n. 356 e 357 da Agência Nacional de Vigilância Sanitária pelas farmácias de manipulação.

Conclui-se, ainda, que a maior parte das formulações contendo $H$. perforatum foi dispensada na forma de extrato seco padronizado em comparação com a dispensação da droga pulverizada. Tal ocorrência é importante uma vez que a garantia de resultado eficaz para os pacientes depressivos depende da padronização na concentração de substâncias consideradas essencialmente importantes para a ação antidepressiva.

Os resultados deste estudo, aliados aos dados obtidos na literatura, indicam que a comunidade médica do município de Anápolis - GO vem buscando uma droga alternativa que seja eficaz e com menos efeitos colaterais (em comparação à utilização de antidepressivos sintéticos) no tratamento ambulatorial da depressão leve a moderada. Mas, é necessário ressaltar que, apesar de não ter sido analisada a ocorrência de utilização de outros medicamentos pelos pacientes para os quais o $\mathrm{H}$. perforatum foi prescrito, a presença de prescrições do $H$. perforatum em associação a outros fitoterápicos representa risco potencial de interação medicamentosa que pode comprometer a efetividade dos tratamentos e colocar em risco a saúde dos usuários. Denotam, portanto a necessidade de empreendimento de programas de educação direcionados aos profissionais da saúde a fim de que assumam o conceito de fitoterápico tal como o de medicamento e contribuam de forma comprometida e responsável para a promoção do uso racional deste fitoterápico.

Recomenda-se a realização de mais estudos que investiguem o uso concomitante de $H$. perforatum e outros medicamentos (prescritos por profissionais médicos ou automedicados) a fim de fornecer informações que contribuam para o uso seguro deste fitoterápico.

\section{REFERÊNCIAS BIBLIOGRÁFICAS}

ALONSO, J. R. Tratado de fitomedicina: bases clínicas e farmacológicas. Buenos Aires: Editora Isis, 1998.

ANDREATINI, R. Uso de fitoterápicos em psiquiatria. Revista Brasileira de Psiquiatria. v. 22, n. 3, p. 104-105, 2000.

BAHLS, S. Tratamento fitoterápico da depressão. Jornal Brasileiro de Psiquiatria. v. 50, n.11-12, p. 389-396, 2001.

BALLONE, G. J. Fitoterápicos [on line]. Disponível: http://www.psiqweb.med.fr/fármaco/fitot.html [capturado em 21 abr. 2005].

BRASIL. Ministério da Saúde. Agência Nacional de Vigilância Sanitária. Resolução Específica - RE n. 356 de 28 de fevereiro de 2002. Determina como medida de interesse sanitário, a apreensão, em todo território nacional, de qualquer produto farmacêutico a base de Kava-Kava (Piper methysticum L.) que não possuam tarja vermelha contendo os dizeres "Venda sob prescrição médica",ou que não possuam registro na Anvisa [on line]. Brasília, 2002a. Disponível: http://www.anvisa.gov.br [capturado em 20 abr. 2006].

BRASIL. Ministério da Saúde. Agência Nacional de Vigilância Sanitária. Resolução Específica - RE n. 357 de 28 de fevereiro de 2002. Determina como medida de interesse sanitário, a apreensão, em todo território nacional, de qualquer produto farmacêutico a base de Erva de São João (hypericum perforatum) que não possuam tarja vermelha contendo os dizeres "Venda sob prescrição médica", ou, que não possuam registro na Anvisa [on line]. Brasília, 2002b. Disponível: http://www.anvisa.gov.br [capturado em 20 abr. 2006].

BRASIL. Ministério da Saúde. Portaria n. 971, de 3 de maio de 2006. Aprova a Política Nacional de Práticas Integrativas e Complementares (PNPIC) no Sistema Único de Saúde. Brasília, 2006a.

BRASIL. Ministério da Saúde. Decreto n. 5.813, de 22 de junho de 2006. Aprova a Política de Plantas Medicinais e Fitoterápicos e dá outras providências. Brasília, 2006b.

CAMPOS, D. S. Medicamentos, plantas medicinales y productos naturales. Fármacos. v. 16, n.1-2, p. 13-20, 2003.

CASS, H. Erva de são joão: o antidepressivo natural. São Paulo: Editora Madras, 1999.

CONSELHO REGIONAL DE FARMÁCIA DO ESTADO DE GOIÁS. Contato e-mail: crfgo@crfgo.org.br. Disponível: http://www.crfgo.gov.br [capturado em 27 abr. 2006]. 
CORDEIRO, C. H. G.; CHUNG, M. C.; SACRAMENTO, L. V. S. Interações medicamentosas de fitoterápicos e fármacos: Hypericum perforatum e Piper methysticum. Revista Brasileira de Farmacognosia. v. 15, n.3, p. 272278, 2005.

D'IPPOLITO, J. A. C.; ROCHA, L. M.; SILVA, R. F. Fitoterapia Magistral: um guia prático para a manipulação de fitoterápicos. São Paulo: Editora Elbergráfica, 2005.

GRÖNING, R.; BREITKREUTZ, J; MÜLLER, R. S. Physico-chemical interactions between extracts of Hypericum perforatum L. and drugs. European Journal of Pharmaceutics and Biopharmaceutics. v. 56, p. 231 - 236, 2003.

KARHOVA, M. et al. Interaction of Hypericum perforatum (St. John's wort) with cyclosporin A metabolism in patient after liver transplantation. Journal of Hepatology. v. 33, p. $853-855,2000$.

KERWIN, R. et al. As drogas e o sistema nervoso. In: PAGE, C. P. et al. Farmacologia Integrada. São Paulo: Editora Manole, 2004.

LIMA, I. V. M.; SOUGEY, E. B.; VALLADA FILHO, H. P. Farmacogenética do tratamento da depressão: busca de marcadores moleculares de boa resposta aos antidepressivos. Revista de Psiquiatria Clínica. v. 31, n. 1, p. $1893-$ 1897, 2004.

LINDE, K. et al. St. John's wort for depression: an overview and meta-analysis of randomized clinical trials. Brazilian Medical Journal v. 313, n. 7052, p. 253-258, 1996.

MANNEL, M. Drug interactions with St John's wort: mechanisms and clinical implications. Drug Safety. v. 27, n. 11, p. 773-797, 2004.

MELEIRO, A. M. A. S. Tratamento da depressão leve a moderada: avaliação da eficácia e segurança do extrato LI 160 S de Hypericum perforatum. Revista Brasileira Médica. v. 57, n. 11, p. 1313-1323, 2000.

MIRANDA-SCIPPA, Â. M. A.; OLIVEIRA, I. R. Antidepressivos. In SILVA, P. Farmacologia. 6. ed. Rio de Janeiro: Editora Guanabara Koogan, 2002.

MURPHY, P. A. St. John's wort and oral contraceptives: reasons for concern? Journal of Midwifery \& Women's Health. v. 47, n. 6, p. $447-450,2002$.

OMS. Alerta de la Organizacion Mundial de la Salud: $H$. perforatum interacción con medicamentos. Boletim informativo de medicamentos, Santiago do Chile, v. 17, n. 12, p. 12-13, 2000.

PITLIUK; R. Depressão, seu tratamento e medicamentos utilizados [on line]. Disponível: http://www.mentalhelp.com.br.htm [capturado em 27 dez. 2005].

RANG, H. P. et al. Fármacos usados nos distúrbios afetivos. In RANG, H. P. et al. Farmacologia. 5. ed. Rio de Janeiro: Editora Elsevier, 2003.

RATES, S. M. K. Promoção do uso racional de fitoterápicos: uma abordagem no ensino de Farmacognosia. Revista Brasileira de Farmacognosia. v. 11, n. 2, p. 57-69, 2001.

SACRAMENTO, E. F.; SILVA, B. B. Vitaminas e minerais. In SILVA, P. Farmacologia. 6. ed. Rio de Janeiro: Editora Guanabara Koogan, 2002.

SCHULZ, V.; HÄNSEL, R.; TYLER, V. E. Fitoterapia Racional: um guia de fitoterapia para as ciências da saúde. 4 ed. São Paulo: Editora Manole, 2002.

SILVEIRA, M. A. B. Ansiolíticos. In SILVA, P. Farmacologia. 6. ed. Rio de Janeiro: Editora Guanabara Koogan, 2002.

SINGH, Y. N. Potential for interaction of kava and St. John's wort with drugs. Journal of Ethnopharmacology. v. 100, p. 108-113, 2005.

TAVARES JÚNIOR, H. Erva-de-são-joão: um novo antidepressivo? Revista de Psiquiatria Clínica. São Paulo, v. 26, n. 3, p. 54-55, 1999. 
WANNMACHER, L. Fármacos usados nos distúrbios afetivos. In FUCHS, F. D; WANNMACHER, L.; FERREIRA, M.B.F. Farmacologia Clínica. 3. ed. Rio de Janeiro: Editora Guanabara Koogan, 2004.

WOLD, R. S. et al. Increasing trends in elderly person's use of nonvitamin, nonmineral dietary supplements and concurrent use of medications. Journal of the American Dietetic Association. v. 105, n. 1, p. 54-63, jan, 2005.

WORLD HEALTH ORGANIZATION. Monographs on selected medicinal plants. Geneva: World Health Organization, 2002. 2 v. 